\title{
A NOTE ON ILL-POSEDNESS OF THE CAUCHY PROBLEM FOR HEISENBERG WAVE MAPS
}

\author{
LUCA CAPOGNA AND JALAL SHATAH
}

(Communicated by David S. Tartakoff)

\begin{abstract}
We introduce a notion of wave maps with a target in the subRiemannian Heisenberg group and study their relation with Riemannian wave maps with range in Lagrangian submanifolds. As an application we establish existence and eventually ill-posedness of the corresponding Cauchy problem.
\end{abstract}

\section{INTRODUCTION}

In the last two decades there have been many contributions to the study of wave maps (see [16] and the references therein). In general, well posedness for the Cauchy problem holds under the condition that the target manifold has bounded geometry. In the present paper we study a simple, explicit model of singular target space, the Heisenberg group endowed with a Carnot-Carathéodory metric. In this setting there are no bounds (even local) on the geometry, but there is a rich and interesting geometric structure which arises in many applications ranging from biology, control theory and physics to several complex variables and PDE (see [4] and the references therein).

The geometric setting. The Heisenberg group $\mathbb{H}^{k}$ is a simply connected, analytic Lie group, whose Lie algebra $\mathfrak{h}=\mathbb{C}^{k} \times \mathbb{R}$ is endowed with the bracket relation $\left[X_{i}, Y_{j}\right]=-4 \delta_{i j} T$ and $\left[X_{i}, T\right]=\left[Y_{i}, T\right]=0$ for any $i, j=1, \ldots, k$; here the set $\left\{X_{i}, Y_{i}, i=1, \ldots, k\right.$ and $\left.T\right\}$ is a vector basis of $\mathfrak{h}$. Using exponential coordinates and the Baker-Campbell-Hausdorff formula (see [4]) one can define the group law on $\mathbb{H}^{k}$ as $(z, t)\left(z^{\prime}, t^{\prime}\right)=\left(z+z^{\prime}, t+t^{\prime}+2 \operatorname{Im}\left(z \bar{z}^{\prime}\right)\right)$, for any $(z, t),\left(z^{\prime}, t^{\prime}\right) \in \mathbb{H}^{k}$. Here $z, z^{\prime} \in \mathbb{C}^{k}, z=\left(z^{1}, \ldots, z^{k}\right), z^{\prime}=\left(\xi^{1}, \ldots, \xi^{k}\right), z \bar{z}^{\prime}=\sum_{j=1}^{k} z^{j} \overline{\xi^{j}}$, and $t, t^{\prime} \in \mathbb{R}$. The vectors $X_{i}, Y_{i}$, and $T$ can be represented in exponential coordinates as the left invariant vector fields $X_{i}=\partial_{x^{i}}+2 y^{i} \partial_{t}, Y_{j}=\partial_{y^{j}}-2 x^{j} \partial_{t}$, and $T=\partial_{t}$, where we denote $(z, t)=\left(x^{1}, \ldots, x^{k} ; y^{1}, \ldots, y^{k} ; t\right)$. For a more detailed study of the Heisenberg group see [8] and [12]. The contact form $\lambda=d t-2 \sum_{i=1}^{m}\left(y^{i} d x^{i}-x^{i} d y^{i}\right)$ on $\mathbb{C}^{k} \times \mathbb{R}$

Received by the editors September 19, 2006.

2000 Mathematics Subject Classification. Primary 35L55, 53C17.

Key words and phrases. Wave maps, Heisenberg group.

The first author was partially supported by a National Science Foundation CAREER grant and by an Arkansas Science and Technology Authority grant.

The second author was partially supported by the National Science Foundation grant DMS 0203485 . 
gives rise to a non-integrable hyperplane distribution

$$
H(z, t)=\operatorname{Kernel}(\lambda(z, t))=\operatorname{Span}\left[X_{1}, \ldots, X_{k}, Y_{1}, \ldots, Y_{k}\right],
$$

the so-called horizontal structure (see [1]). In view of non-integrability every two points in $\mathbb{H}^{k}$ can be joined by a smooth curve $\gamma: I \rightarrow \mathbb{H}^{k}$ such that $\gamma^{\prime}(s) \in H(\gamma(s))$ for any $s \in I$, and $I$ an interval (such curves are called horizontal curves). The Carnot-Carathéodory (CC) metric (see [11]) is defined as follows: For any $\delta>0$, and $P, Q \in \mathbb{H}^{k}$ we define the set $A(\delta)$ that includes all absolutely continuous curves $\gamma:[0, \delta] \rightarrow \mathbb{H}^{k}$ such that $\gamma(0)=P, \gamma(\delta)=Q$, and $\gamma^{\prime}=\sum_{i=1}^{k}\left(a_{i} X_{i}+b_{i} Y_{i}\right) \in$ $H(\gamma(s))$ for almost every $0 \leq s \leq \delta$, and $\left|a_{i}\right|,\left|b_{i}\right| \leq 1$. The CC distance between $P$ and $Q$ is defined to be $d_{C}(P, Q)=\inf \{\delta>0 \mid A(\delta) \neq \emptyset\}$. The length of a horizontal curve $\gamma:[0,1] \rightarrow \mathbb{H}^{k}$ (in the metric space sense) is given by $\int_{0}^{1} \sum_{i=1}^{k}\left|\Pi_{z^{i}}\left(\gamma^{\prime}\right)\right| d s$, where $\Pi_{z^{i}}: \mathbb{H}^{k} \rightarrow \mathbb{C}$ denotes the orthogonal projection onto the $z^{i}=\left(x^{i}, y^{i}\right)$ variable.

Riemannian approximation. Following Koranyí [12] and Gromov [11], we recall that, as a metric space, $\left(\mathbb{H}^{k}, d_{C}\right)$ is the Gromov-Hausdorff limit of $\left(\mathbb{R}^{2 k+1}, d_{L}\right)$ as $L \rightarrow \infty$, where $d_{L}$ denotes the distance function associated to a sequence of Riemannian metrics $g_{L}$. To be more specific: If $L>1$ we define the metric $g_{L}$ on $\mathbb{R}^{2 k+1}$, so that the left invariant basis of $\mathbb{H}^{k}$ given by $X_{i}, Y_{i}, i=1, \ldots, k$, and $T=L^{-\frac{1}{2}} \partial_{t}$ is orthonormal. If $u=(x, y, t): \mathbb{R}^{1+l} \rightarrow \mathbb{H}^{k}$, then for $i=0,1, \ldots, l$ $u_{*} e_{i}=\sum_{j=1}^{k}\left(\partial_{i} x^{j}\right) X_{j}+\left(\partial_{i} y^{j}\right) Y_{j}+u^{*} \lambda\left(e_{i}\right) T$, where $u^{*} \lambda\left(e_{i}\right)=\partial_{i} t-2\left(y \partial_{i} x-x \partial_{i} y\right)$, and $\left\{e_{i}\right\}$ is the canonical basis of $\mathbb{R}^{1+l}$. Consequently one has

$$
\sum_{i=0}^{l}\left\|u_{*} e_{i}\right\|_{g_{L}}^{2}=\sum_{i=0}^{l}\left\langle g_{L} u_{*} e_{i}, u_{*} e_{i}\right\rangle=\left|\nabla_{\mathbb{R}^{1+l}} z\right|^{2}+L\left|\partial_{i} t-2\left(y \partial_{i} x-x \partial_{i} y\right)\right|^{2} .
$$

The Riemannian curvature tensor corresponding to $g_{L}$ is unbounded (from above and from below) as $L \rightarrow \infty$ (see [4] for details on the blow-up). In [12], A. Koranyì showed that length minimizing curves with respect to the Carnot-Caratheodory metric on $\mathbb{H}^{k}$ can be obtained as a limit of the geodesics in $\left(\mathbb{C}^{k} \times \mathbb{R}, g_{L}\right)$. In this paper we indirectly provide a different proof of this convergence.

Heisenberg wave maps and the Cauchy problem. Wave maps are critical points of an action functional. Such action is modeled, roughly speaking, on the energy corresponding to the metric on the manifold. The "energy" of a map with target in $\left(\mathbb{H}^{k}, d_{C}\right)$ may be defined adapting a construction in [13], where a notion of energy is given for maps from a manifold to any metric space. In our case the energy can be computed explicitly: If $u=(z, t): \mathbb{R}^{l} \rightarrow \mathbb{H}^{k}$ is a weakly differentiable map, then

$$
E(u)= \begin{cases}\int_{\mathbb{R}^{l}}|\nabla z|^{2} d p, & \text { if } t_{i}=\sum_{j=1}^{k} \operatorname{Im}\left(z^{j} \bar{z}_{i}^{j}\right), i=1, \ldots, l, \text { a.e. in } \mathbb{R}^{l}, \\ \infty, & \text { otherwise }\end{cases}
$$

(see [5]). This definition is exactly what one would expect in view of (1.2), namely finite energy maps are tangent a.e. to the horizontal hyperplane distribution $H(p)$ defined in (1.1). Equivalently, by the Frobenius Theorem, we can say that $u=(z, t)$ has finite energy if and only if $\int|\nabla z|^{2}<\infty$, and $\sum_{l=1}^{k} \operatorname{Im}\left(\partial_{i} z^{l} \partial_{j} \bar{z}^{l}\right)=$ 0 , for any $i, j=1, \ldots, l$. In other words, the map $z: \mathbb{R}^{n} \rightarrow \mathbb{C}^{k}$ is weakly anisotropic (if $l=k$, then the map is called Lagrangian). 
The action functional corresponding to $E(u)$ is defined for all finite energy maps $u=(x+\boldsymbol{i} y, t)=(z, t): R^{1+l} \rightarrow \mathbb{H}^{k}$ as

$$
A(u)=\int_{\mathbb{R}^{l} \times \mathbb{R}}\left|\partial_{0} z\right|^{2}-\sum_{j=1}^{n}\left|\partial_{j} z\right|^{2} .
$$

Formally, ${ }^{1}$ we can define Heisenberg wave maps as critical points of $A(u)$ in the space of maps which satisfy the Lagrangian constraints. The corresponding Cauchy problem consists in finding those Heisenberg wave maps which satisfy the initial conditions $u_{\left.\right|_{\text {initial time }}}=h$, and $\partial_{0} u_{\left.\right|_{\text {initial time }}}=g$, where $h: \mathbb{R}^{l} \rightarrow \mathbb{H}^{k}$ is ${ }^{2}$ a horizontal map, and $g: \mathbb{R}^{l} \rightarrow \mathbb{R}^{2 k+1}$ is such that $g \in H(h)$.

If we interpret the solution of the Cauchy problem for the critical points of the action (1.3) as a vibrating "horizontal" membrane, then in our setting the membrane is a subset of the Heisenberg group, "tangent" a.e. to the horizontal hyperplanes $H(p)$, and the vibrations have to occur along the horizontal directions.

Results and comments. The main results in this paper concern the construction of Heisenberg wave maps and the corresponding Cauchy problem. The difficulties stem from the non-linearity and the non-holonomic nature of the Legendrian (or Lagrangian) constraints. We show that Riemannian wave maps with a target in a Lagrangian manifold, satisfying a suitable "rank condition", are Heisenberg wave maps. This gives us a tool to construct Heisenberg wave maps and solve the Cauchy problem. The new feature in these results is that in general the Cauchy problem is not locally well posed, even for smooth initial data with arbitrarily small norm. In fact, we construct examples of distinct Heisenberg wave maps satisfying the same initial, smooth, conditions. In a certain sense (to be made precise later) these initial data are "degenerate". Such a lack of uniqueness is not entirely surprising in view of the well known corresponding phenomena for geodesics (see [12] and [11]). Our examples can also be used to show the lack of global well-posedness, even for non-degenerate initial data, in the $1+1$ dimensional case.

The following is a "sample" result, following from our work.

Theorem 1.1. The Cauchy problem for Legendrian wave maps $u: \mathbb{R}^{1+k} \rightarrow \mathbb{H}^{k}$ is locally well-posed in the space

$$
C\left([0, T), H^{s}\left(\mathbb{R}^{k}\right)\right) \times C\left([0, T), H^{s-1}\left(\mathbb{R}^{k}\right)\right), \text { with } s>\frac{k}{2}+1,
$$

and for a rank $k$ initial position. The time $T>0$ depends on the initial data (not only on their Sobolev norm).

The relatively high regularity requested of the initial data (in comparison with the Riemannian setting) is due to the nature of the Euler-Lagrange equations. The latter reduces to a coupled system of quasilinear hyperbolic equations and transport equations whose coefficients depend on the solutions of the hyperbolic equations. Transport equations with rough coefficients have been studied (see for instance the papers of DiPerna-Lions [7], Bahouri-Chemin [3] and Ambrosio [2]). However, unless special structure conditions are present, in order to have existence and uniqueness results one needs at least $L^{1}\left([0, T), L^{\infty}\right)$ regularity for the coefficients

\footnotetext{
${ }^{1}$ At the end of Section 2 we define Heisenberg wave maps to be solutions of the Euler-Lagrange system.

${ }^{2}$ We refer to $h$ as an initial position.
} 
and their derivatives. To achieve such regularity we have to restrict our hypothesis to rather smooth initial data. This situation has an analogue in the study of the Vlasov-Maxwell system (see [9], and [10]), where coupled systems of wave equations and transport equations appear as well.

Since neither smoothness nor small norm of initial data grants well-posedness, we chose not to put any emphasis on the question of sharpest regularity assumption. In conclusion we remark that the action functional corresponding to the Riemannian metrics $g_{L}$ can be easily computed and is essentially (1.3) with an additional penalization term which measures the "size" of the non-horizontal component of the differential of the map. It is then natural to conjecture that critical points of the penalized action (i.e. the $g_{L}$-wave maps) converge to Heisenberg wave maps. We think that such convergence does not hold because of the presence of rapidly oscillating contributions due to non-horizontal terms; however we are unable to find a counterexample, and this remains a challenging open problem. In this regard we want to emphasize the similarities between our problem and the work of Zeng and one of us in [17], in which the Riemannian wave map is obtained by adding an order zero penalization term ${ }^{3}$ to the action functional. In [17] it is shown that the free "penalized" system converges to the (constrained) Riemannian wave map system. The approach in [17] fails in our setting because the constraints (i.e. our penalization term) are of order one (that is, they involve first order derivatives of the map). In the next section we will give more details on this topic.

Notation. As usual, when considering partial derivatives in the Minkowski space $\mathbb{R}^{1+l}$, we will denote by $\partial_{0}$ the time derivative, and by $\partial_{1}, \ldots, \partial_{l}$ the space derivatives. The time variable is denoted by either $s_{0}$ or $\tau$, while the space variables are denoted by $s_{1}, \ldots, s_{l}$. Whenever $z, z^{\prime} \in \mathbb{C}^{m}$ we will let $z z^{\prime}=\sum_{i=1}^{m} z^{i}\left(z^{\prime}\right)^{i}$.

\section{Euler-Lagrange EQUATIONS}

In this section we formally derive the Euler-Lagrange equations for the Heisenberg wave maps. We will use the method of Lagrange multipliers. Let $\vec{\phi}=$ $\left(\phi^{0}, \ldots, \phi^{l}\right) \in C^{\infty}\left(\mathbb{R}^{l+1}, \mathbb{R}^{l+1}\right)$ be a vector-valued parameter function and consider the expression

$$
\begin{aligned}
\sum_{j=1}^{k} \int_{\mathbb{R}^{l} \times \mathbb{R}} \frac{1}{2} & \left(\left|\partial_{0} z^{j}\right|^{2}-\sum_{i=1}^{l}\left|\partial_{i} z^{j}\right|^{2}\right) d s d v \\
& +\sum_{i=0}^{l} \int_{\mathbb{R}^{l} \times \mathbb{R}} \phi^{i}(v, s)\left[\partial_{i} t-2 \sum_{j=1}^{k} \operatorname{Im}\left(z^{j} \partial_{i} \bar{z}^{j}\right)\right] d v d s .
\end{aligned}
$$

Taking variations of $(2.1)$ in the variables $t$ and in $z^{j}, j=1, \ldots, k$, we obtain

$$
-\left(\partial_{0}^{2} z^{j}-\Delta z^{j}\right)+4 i \sum_{i=0}^{l}\left(\phi^{i} \partial_{i} z^{j}+\partial_{i} \phi^{i} z^{j}\right)=0 \quad \text { and } \sum_{i=1}^{l} \partial_{i} \phi^{i}+\partial_{0} \phi^{0}=0,
$$

\footnotetext{
${ }^{3}$ The penalization term does not involve derivatives of the map; it is in fact the distance function from the manifold multiplied by $L$.
} 
where $\Delta=\sum_{i=1}^{l} \partial_{i}^{2}$ is the space-Laplacian. Combining the two expressions above we obtain the Euler-Lagrange PDE for Legendrian wave maps: For $i=0, \ldots, l$,

$$
\partial_{i} t-2 \sum_{j=1}^{m} \operatorname{Im}\left(z^{j} \partial_{i} \bar{z}^{j}\right)=0, \text { and } \begin{cases}\square \vec{z}-4 i \sum_{l=0}^{l} \phi^{i} \partial_{i} \vec{z} & =0 \\ \operatorname{div} \vec{\phi} & =0\end{cases}
$$

This is a system of $2 k+l+2$ partial differential equations, with $2 k+l+2$ unknowns $\left(z_{1}, \ldots, z_{k}, t, \phi^{0}, \ldots, \phi^{l}\right)$, and it generalizes the geodesics equations in $\mathbb{H}^{k}$ (which can be recovered from (2.2) by imposing constant initial data and no dependence on $v$ ).

The equations derived through the Lagrange multipliers method can be rigorously interpreted as Euler-Lagrange equations of the variational problem only if the map is at least $C^{2}$ smooth. This can be verified with a simple computation of the differential of the action and with standard functional analysis techniques.

If we assume $H^{1} \cap L^{\infty}$ regularity for $(z, t)$ and $\phi$, then the system (2.2) may be interpreted in a weak sense. Under the same regularity assumptions and arguing as in [5], the Legendrian constraints in (2.2) can be reformulated in terms of the equivalent "Lagrangian" conditions:

$$
\sum_{j=1}^{k} \operatorname{Im}\left(\partial_{i} z^{j} \partial_{l} \bar{z}^{j}\right)=0, \quad i, l=0, \ldots, l .
$$

The difficulty of the problem and the degenerate character of the PDE stem from the non-linear nature of the derivative constraints in (2.2) and (2.3).

Euler-Lagrange equations in the Riemannian approximation. We derive the Euler Lagrange equations for the approximating metrics $g_{L}$. The structure of such equations bears a striking resemblance to the structure of the system (2.2), suggesting that the latter may also hold with relaxed smoothness hypothesis.

Using (1.2), we can compute the action functional for the metrics $g_{L}$, associated to maps $u: \mathbb{R}^{1+l} \rightarrow \mathbb{H}^{k}$,

$$
\begin{aligned}
A_{L}(u)= & \int_{\mathbb{R}^{1+l}}\left|\partial_{0} z\right|^{2}-\left|\nabla_{\mathbb{R}^{l}} z\right|^{2} d p \\
& +L \int_{\mathbb{R}^{1+l}}\left[\left(\partial_{0} t-2 \operatorname{Im}\left(z \partial_{0} \bar{z}\right)\right)^{2}-\sum_{i=1}^{l}\left(\partial_{i} t-2 \operatorname{Im}\left(z \partial_{i} \bar{z}\right)\right)^{2} d p\right]
\end{aligned}
$$

Next we take variations in $z$ of (2.4), obtaining

$$
\begin{aligned}
\int_{R^{1+l}}\{ & \partial_{0} z \cdot \partial_{0} \psi-\sum_{i=1}^{l} \partial_{i} z \cdot \partial_{i} \psi \\
& \left.\quad-2 L\left[\lambda^{0}\left(\boldsymbol{i} \partial_{0} z \cdot \phi-(\boldsymbol{i} z) \cdot \partial_{0} \phi\right)-\sum_{i=1}^{l} \lambda^{i}\left(\boldsymbol{i} \partial_{i} z \cdot \phi-(\boldsymbol{i} z) \cdot \partial_{i} \phi\right)\right]\right\} d p
\end{aligned}
$$

where we have let $\lambda^{i}=\partial_{i} t-2 \operatorname{Im}\left(z \partial_{i} \bar{z}\right)$, for $i=0,1, \ldots, l$. The variation in $t$ yields the equation $\int_{R^{1+l}}\left(\lambda^{0} \partial_{0} \psi-\sum_{i=1}^{l} \lambda^{i} \partial_{i} \psi\right) d p=0$. 
Integrating by parts and using the latter equality in (2.5) we obtain the system of $2 k+1$ equations in $2 k+1$ unknowns $(z, t)$,

$$
\left\{\begin{array}{l}
\square \vec{z}-4 i L\left(\lambda^{0} \partial_{0} \vec{z}-\sum_{i=1}^{l} \lambda^{i} \partial_{i} \vec{z}\right)=0, \\
\partial_{0} \lambda^{0}-\sum_{i=1}^{l} \partial_{i} \lambda^{i}=0 .
\end{array}\right.
$$

Comparing (2.2) and (2.6) we see that both systems are of the form $\square \vec{z}$ equal to the inner product of a divergence free vector field and a gradient. Let us sketch how, using standard energy methods (see [16] and [15]), one can verify that solutions $z_{L}$ to (2.6) converge weakly in $H^{1}$ to a Legendrian map: Let $u_{L}=(z, t)$ be a solution of (2.6). Since $u_{L}$ is a Riemannian wave map, then it satisfies the standard energy identity (see [16, page 82])

$$
E_{L}\left(u_{L}(\tau)\right)=\frac{1}{2}\left\|D u_{L}(\tau)\right\|_{L^{2}\left(\mathbb{R}^{l}\right)}^{2}=\frac{1}{2}\left\|D u_{L}(0)\right\|_{L^{2}\left(\mathbb{R}^{l}\right)}^{2}, \text { for any time } \tau>0 .
$$

Here, the energy density is given by (1.2),

$$
\begin{aligned}
\left|D u_{L}(\tau)\right|=\sum_{a, b=1}^{2 k+1} \sum_{0=1}^{l}\left(g_{L}\right)_{a b} & {\left[\partial_{i} u^{a} \partial_{i} u^{b}\right] } \\
& =\left[\left|\nabla_{\mathbb{R}^{l+1}} z\right|^{2}+L \sum_{i=1}^{l}\left(\partial_{i} t-2 \operatorname{Im}\left(z \partial_{i} \bar{z}\right)\right)^{2}\right] .
\end{aligned}
$$

If we choose initial data of the form

$$
\left.u_{L}\right|_{\tau=0}=h \in C^{2}\left(\mathbb{R}^{l}, \mathbb{R}^{2 k+1}\right) \text { horizontal map, and }\left.\partial_{0} u\right|_{\tau=0}=g+\frac{c}{L} T,
$$

where $g \in C^{1}\left(\mathbb{R}^{l}, \mathbb{R}^{2 k+1}\right) \cap L^{2}$ satisfies $g(s) \in H(h(s))$, for $s \in \mathbb{R}$, and $c \in C^{1}\left(\mathbb{R}^{l}, \mathbb{R}\right) \cap$ $L^{2}$, then the energy at time $\tau=0$ will be

$$
E\left(u_{L}(0)\right)=\left\|g+\frac{c}{L}\right\|_{L^{2}\left(\mathbb{R}^{l}\right)}^{2}+\|\nabla h\|_{L^{2}\left(\mathbb{R}^{l}\right)}^{2}+\|c\|_{L^{2}\left(\mathbb{R}^{l}\right)}^{2} \leq C(g, c, h) ;
$$

note that $C$ does not depend on $L$. Hence the energy identity yields a uniform $H^{1}$ bound on $u_{L}$, hence the weak convergence to a Legendrian map. If $l=1$, then one has actually pointwise bounds on the energy density and its derivatives (see [15]), and consequently the convergence is uniform.

Both for $l=1$ and in higher dimensions, the weak form of convergence we are able to obtain is not sufficient to pass to the limit and conclude that (2.2) is the Euler-Lagrange system for our problem.

Heisenberg wave maps. In the rest of the paper, we will call a Heisenberg wave map any map $z: \mathbb{R}^{1+l} \rightarrow \mathbb{C}^{k}$ for which we can find $\phi: \mathbb{R}^{1+l} \rightarrow \mathbb{R}^{1+l}$ so that $(2.2)$ and the constraints (2.3) are satisfied (in a weak sense).

Once we have the $z$ component for wave maps in the Heisenberg groups, then we can construct the whole map by means of a Legendrian lift. The Legendrian lift of a map $z: \mathbb{R}^{1+1} \rightarrow \mathbb{C}$ is a map $(z, t): \mathbb{R}^{1+1} \rightarrow \mathbb{H}^{1}$ which is Legendrian and which projects to $z$. Such lift is obtained by "lifting", for each $s \in \mathbb{R}$, the curve $\tau \rightarrow z(\tau, s)$, with end-point along the initial data $z(0, s)$ (see for instance [11], and [4]). Analogue considerations hold in higher dimensions. 


\section{Comparing Riemannian and Heisenberg wave maps}

In this section we study the link between Riemannian and the Heisenberg wave maps. We start by giving a characterization for any smooth map to be a Heisenberg wave map.

Proposition 3.1. Let $z: \mathbb{R}^{l+1} \rightarrow \mathbb{C}^{k}$ be a smooth map, and let $M$ denote the range of $z$. For any $p=z(\tau, s) \in M$ set $T_{p} M$ to be the range of $d_{(\tau, s)} z, T M$ is the corresponding bundle and $m=m(p)$ is the dimension of $T_{p} M$. If the following conditions hold:

(i) $\square z \in i T M$ in a neighborhood of $p$,

(ii) $\quad M$ is a manifold in a neighborhood of $p$, and $m \leq l$,

(iii) $\quad$ iT $M \perp T M$,

then $z$ is a Heisenberg wave map in a neighborhood of $p$.

Proof. Condition (ii) grants the existence of linearly independent, smooth vector fields $\left\{\partial_{l 1} z, \ldots, \partial_{l_{m}} z\right\}$, which generate $T M$ in a neighborhood of $p$. Since $m \leq l$, at least one index is missing from this list, and we will denote its corresponding direction by $\partial_{\alpha_{0}} z \in T M$. We can find smooth functions $c_{b}$, such that

$$
\partial_{\alpha_{0}} z-\sum_{b=1}^{m} c_{b} \partial_{l_{b}} z=0,
$$

in a neighborhood of $p$.

On the other hand, $(i)$ guarantees the existence of smooth functions $\psi_{b}$ such that $\square z-4 i \sum_{b=1}^{m} \psi_{b} \partial_{l_{b}} z=0$. Hence, for any smooth function $\lambda$ we have $\square z-$ $4 \boldsymbol{i} \sum_{b=1}^{m} \psi_{b} \partial_{l_{b}} z-4 \boldsymbol{i} \lambda\left(\partial_{\alpha_{0}} z-\sum_{b=1}^{m} c_{b} \partial_{l_{b}} z\right)=0$. The latter suggests an obvious choice for $\phi$. To conclude, we only need to choose $\lambda$ to be a solution of the transport equation

$$
\partial_{\alpha_{0}} \lambda+\sum_{b=1}^{m}\left(\psi_{b}-c_{b} \lambda\right)=0 .
$$

Theorem 3.2. Let $z: \mathbb{R}^{l+1} \rightarrow \mathbb{C}^{k}$ be a smooth map. Define $M, T M$, and $m$ as in Proposition 3.1. If we assume that $M$ is a Lagrangian manifold and $m \leq l$, then $z$ is a Heisenberg wave map if and only if it is a Riemannian wave map into $M$.

Proof. If $z: \mathbb{R}^{1+l} \rightarrow M$ is a smooth Riemannian wave map, then $\square \vec{z} \perp T M$. Consequently, since $M$ is Lagrangian, both $(i)$ and (iii) in Proposition 3.1 hold, and $z$ is a Heisenberg wave map. Vice versa, if $z: \mathbb{R}^{l+1} \rightarrow \mathbb{C}^{k}$ is a smooth Heisenberg map, then $\square \vec{z} \in \operatorname{span}\left\{\boldsymbol{i} \partial_{\alpha} \vec{z} \mid \alpha=0, \ldots, l\right\}$. In case $M$ is Lagrangian, then this implies $\square \vec{z} \perp T M$, hence $z$ is a Riemannian wave map.

Several remarks are in order:

Theorem 3.2 extends immediately to $C^{1}$ maps. The only substantial difference is in solving (3.3), because now the coefficients of this transport equation will not be smooth. If $z$ is a $C^{1}$ Riemannian wave map into $M$, then the components of $\vec{c}$ in (3.2) are Lipschitz. The existence of the solution of transport equations with Lipschitz coefficients is standard (see for instance [7, Proposition II.1]).

Let $z: \mathbb{R}^{l+1} \rightarrow \mathbb{C}^{k}$ be a smooth map. Define $M, T M$, and $m$ as in Proposition 3.1. If $M$ is Lagrangian, and $(i),(i i)$ hold, then $z$ is both a Heisenberg wave map and a Riemannian wave map into $M$. 
Let $z: \mathbb{R}^{l+1} \rightarrow \mathbb{C}^{k}$ be a smooth map. Define $M, T M$, and $m$ as in Proposition 3.1 , and assume that $(i)$ and $(i i)$ hold. For any smooth manifold $N \subset \mathbb{C}^{k}$, such that $M \subset N$, and $i T_{p} M \perp T_{p} N$, for all $p \in M$, one has that $z$ is both a Heisenberg wave map and a Riemannian wave map into $N$.

As a converse to the previous remark: Consider a smooth Heisenberg wave map $z: \mathbb{R}^{l+1} \rightarrow \mathbb{C}^{k}$, and define $M, T M$, and $m$ as in Proposition 3.1. For any smooth manifold $N \subset \mathbb{C}^{k}$, such that $M \subset N$, and for every $p \in M, \boldsymbol{i} T_{p} M \perp T_{p} N$, one has that $z$ is a Riemannian wave map into $N$.

The relation between Riemannian wave maps and Heisenberg wave maps depends upon finding a manifold $N$ that satisfies the hypothesis described above. In general there is not a unique choice of such $N$, and this accounts in part for the lack of uniqueness in the Cauchy problem for Heisenberg wave maps.

As a corollary of Theorem 3.2 we can prove Theorem 1.1.

Proof. Since the initial data for $z$ is given by a Lagrangian manifold $M$ we consider the unique Riemannian wave map $z$ with the prescribed initial data. In view of Theorem 3.2, $z$ will also be a Heisenberg map. Short time uniqueness and $H^{s}$ estimates follow from standard hyperbolic PDE theory (see for instance [16]). Note that if the rank of the map drops below $l$, then there may be more than one Lagrangian manifold along which the flow will continue, resulting in non-uniqueness.

\section{Non-uniqueness of the Heisenberg wave map flow}

The maximum rank hypothesis in Theorem 1.1 is necessary. In this section we exhibit examples where this hypothesis fails and one has non-uniqueness for the solutions of the Cauchy problem. This phenomena is reminiscent of the well known lack of uniqueness in the Cauchy problem for geodesics (see [12]). In the first example we actually show that even the maximum rank hypothesis is not enough to guarantee global well posedness, regardless of the smoothness or the size of the initial data. In fact, solutions for smooth initial data exist in a unique fashion for a short period of time, and then they may branch off into infinitely many solutions. This phenomenon does not occur for geodesics, but holds in the $1+l$ dimensional case for $l \geq 1$.

A). We construct infinitely many distinct Heisenberg wave maps $z: \mathbb{R}^{1+1} \rightarrow \mathbb{C}$, with the same initial data. Consider the rank-zero initial data

$$
\left\{\begin{array}{l}
z(0, s)=z_{0} \in \mathbb{C}, \text { for any } s \in \mathbb{R}, \\
\partial_{0} z(0, s)=s \eta_{0}, \text { for a fixed } \eta_{0} \in \mathbb{C}, \text { and for any } s \in \mathbb{R} .
\end{array}\right.
$$

Let $\gamma:[0, \infty) \rightarrow \mathbb{C}$ be a $C^{2}$ curve, parametrized by an arc length satisfying $\gamma(0)=z_{0}$, and $\gamma^{\prime}(0)=\eta_{0}$. Denote by $k=\left|\gamma^{\prime \prime}\right|$ the curvature of $\gamma$. We want to construct Heisenberg wave maps, hence we need to construct maps $z: \mathbb{R}^{1+1} \rightarrow \mathbb{C}$ and divergence free vector fields $\vec{\phi}=\left(\phi^{0}, \phi^{1}\right)$ satisfying (2.2) and (2.3). Since any curve in $\mathbb{C}$ is a Lagrangian curve, the constraint (2.3) is always trivially satisfied. To simplify the computations we will let $\phi^{0}=\partial_{1} f$ and $\phi^{1}=-\partial_{0} f$ for some choice of a smooth function $f$. We look for solutions of the form $z=\gamma(u)$, with $u: \mathbb{R}^{1+1} \rightarrow \mathbb{R}$. Substituting this ansatz in (2.2), we obtain the necessary condition $\square u=0$ and $\left(\partial_{0} u^{2}-\partial_{1} u^{2}\right) k=4\left(\partial_{1} f \partial_{0} u-\partial_{0} f \partial_{1} u\right)$. The latter is satisfied by $u(\tau, s)=s \tau$, once we choose $f(\tau, s)=k(s \tau)\left(s^{2}+\tau^{2}\right)$. 
Since the curve $\gamma$ was chosen arbitrarily, among those satisfying the same initial conditions, we have that there are infinitely many solutions to this Cauchy problem for Heisenberg wave maps.

When evaluated at time $\tau=-1$, the solution $z(-1, s)$ to the Cauchy problem for the initial conditions (4.1) is simply $z(-1, s)=\gamma(-s)$. Its transversal velocity is $\partial_{0} z(-1, s)=-s \gamma^{\prime}(-s)$. Hence, if we start the flow at time $\tau=-1$ with the whole curve $\gamma$, then at time $\tau=0$ the solution will become degenerate (reduced to the point $\gamma(0))$ and the subsequent evolution is not unique. In fact it can continue along any other curve $\gamma_{1}$ which is tangent to $\gamma$ and has the same curvature at the point of degeneracy. It is easy to verify that the map $z(\tau, s)=\gamma(\tau s)$ if $\tau \leq 0$ and $z(\tau, s)=\gamma_{1}(\tau s)$ if $\tau \geq 0$ obtained by gluing at time $\tau=0$ the two Heisenberg wave map flows, continue to solve the system (2.2). As a consequence of this construction the Cauchy problem for the Heisenberg wave maps cannot be globally well posed in dimension $1+1$, even for initial data with maximum rank.

This example is in fact typical of how smooth local solutions can be extended in a non-unique fashion. We can generalize the above example to

Theorem 4.1. Let $\Sigma_{1}$ and $\Sigma_{2}$ be two smooth Lagrangian submanifolds of $\mathbb{C}^{k}$, such that $\Sigma_{1}$ and $\Sigma_{2}$ are tangent of degree two on a $k-1$ dimensional manifold $S$. Given two Riemannian wave maps

$$
z_{i}: \mathbb{R}^{1+k} \rightarrow \Sigma_{i}, \quad \operatorname{Rank}\left(d z_{i}\right)=k, \text { for } i=1,2
$$

with the same smooth initial data on $S$, then

$$
z(\tau, s)= \begin{cases}z_{1}(\tau, s) & \text { for } \tau \leq 0, \\ z_{2}(\tau, s) & \text { for } \tau \geq 0,\end{cases}
$$

is a Heisenberg wave map.

Proof. By Theorem 1.1, both $z_{1}$ and $z_{2}$ are Heisenberg wave maps from a neighborhood of $\mathbb{R}^{k} \times\{0\}$ into $\Sigma_{1}$ and $\Sigma_{2}$, respectively. Thus we only need to show that we can glue the two solutions $z_{1}$ and $z_{2}$ together and still solve the system (2.2). We first observe that since $z_{1}$ and $z_{2}$ are smooth then $d z_{1}=d z_{2}$ and $\partial_{i} d z_{1}=\partial_{i} d z_{2}$, $i=1, \ldots, k$, on $S$. Moreover, using the wave map equation

$$
\partial_{0}^{2} z_{a}-\Delta z_{a}+\Gamma_{a}\left(d z_{a}, d z_{a}\right)=0, \quad a=1,2,
$$

we observe that $\partial_{o}^{2} z_{1}=\partial_{0}^{2} z_{2}$ on $S$ since by hypothesis the Christoffell symbols of $\Sigma_{1}$ and $\Sigma_{2}$ agree on $S$. Thus the map $z(\tau, s)$ defined in $(4.2)$ is $C^{2}$ across $\tau=0$ and smooth elsewhere.

Since $z_{1}$ and $z_{2}$ are Riemannian wave maps then

$$
\square z_{a}=\sum_{b=1}^{k} \lambda_{a}^{b} n_{a}^{b}
$$

where $\left\{n_{b}^{a}\right\}$ is a basis of the normal bundle for $\Sigma_{a}, a=1$, or $a=2$, depending on the sign of $\tau$. Since $\operatorname{rank}\left(d z_{1}\right)=\operatorname{rank}\left(d z_{2}\right)=k$ then there exists $k$ linearly independent vectors $\left\{\partial_{l_{1}} z, \ldots, \partial_{l_{k}} z\right\}$ that span the normal bundle in a neighborhood of $\tau=0$. One index is missing from this list, and we will denote the corresponding vector by $\partial_{\alpha_{0}} z$. There exist $C^{1}$ functions $c_{1}, \ldots, c_{k}$ such that

$$
\partial_{\alpha_{0}} z=\sum_{b=1}^{k} c_{b} \partial_{l_{b}} z \text {. }
$$


Equation (4.3) can then be rewritten as

$$
\square z-4 i \sum_{b=1}^{k} \psi^{l_{b}} \partial_{l_{b}} z=0 .
$$

We need to find a divergence free vector field $\phi$ such that $\square z-4 i \sum_{\alpha=0}^{k} \phi^{\alpha} \partial_{\alpha} z=$ 0 . In view of (4.4) and (4.5), for any function $\lambda$ we have that $\square z-4 i \sum_{b=1}^{k} \psi^{l_{b}} \partial_{l_{b}} z-$ $4 \boldsymbol{i} \lambda\left(\partial_{\alpha_{0}} z-\sum_{b=1}^{k} c_{b} \partial_{l_{b}} z\right)=0$, which yields an obvious choice for $\phi$. The only condition left to verify is that $\partial_{\alpha_{0}} \lambda+\sum_{b=1}^{k} \partial_{l_{b}}\left(\psi^{l_{b}}+\lambda c_{b}\right)=0$. Since this is a nondegenerate transport PDE with $C^{1}$ coefficients we can solve it and find $\lambda$. In this way we obtain $\phi$, completing the proof of the theorem.

\section{REFERENCES}

[1] B. Aebischer, M. Borer, M. Kälin, Ch. Leuenberger and H. M. Reimann, Symplectic Geometry, Progr. Math., 124 (1994), Birkhäuser. MR1296462 (96a:58082)

[2] L. Ambrosio, Transport equation and Cauchy problem for BV vector fields. Invent. Math. 158 (2004), no. 2, 227-260. MR2096794 (2005f:35127)

[3] H. Bahouri and J.-Y. Chemin, Équations de transport relatives á des champs de vecteurs non-lipschitziens et mécanique des fluides (French) [Transport equations for non-Lipschitz vector fields and fluid mechanics]. Arch. Rational Mech. Anal. 127 (1994), no. 2, 159-181. MR1288809 (95g:35164)

[4] L. Capogna, D. Danielli, S. Pauls and J. Tyson, An introduction to the Heisenberg group and the sub-Riemannian isoperimetric problem, Progr. Math., 259, Birkhäuser, Basel, 2007. MR2312336

[5] L. Capogna and F.-H. Lin, Legendrian energy minimizers. I: Heisenberg group target, Calc. Var. Partial Differential Equations 12 (2001), no. 2, 145-171. MR1821235 (2002k:58032)

[6] G. Citti and A. Sarti, A cortical based model of perceptual completion in the roto-translation space. J. Math. Imaging Vision 24 (2006), no. 3, 307-326. MR2235475 (2007b:92016)

[7] R. DiPerna and P.-L. Lions, Ordinary differential equations, transport theory and Sobolev spaces. Invent. Math. 98 (1989), no. 3, 511-547. MR1022305 (90j:34004)

[8] G. B. Folland and E. M. Stein, Hardy Spaces on Homogeneous Groups, Princeton Univ. Press, Princeton; University of Tokyo Press, Tokyo, 1982. MR657581 (84h:43027)

[9] R. T. Glassey, The Cauchy problem in kinetic theory, SIAM, Philadelphia, 1996. MR1379589 (97i:82070)

[10] R. T. Glassey and W. Strauss, Singularity formation in a collisionless plasma could occur only at high velocities, Arch. Rat. Mech. Anal. 92 (1986), 56-90. MR816621 (87j:82064)

[11] M. Gromov, Carnot-Caratheodory spaces seen from within, in Sub-Riemannian geometry, 79-323, Progr. Math., 144, Birkhäuser, Basel, 1996. MR1421823 (2000f:53034)

[12] A. Koranyi, Geometric aspects of analysis on the Heisenberg group. Topics in modern harmonic analysis, Vols. I, II (Turin/Milan, 1982), 209-258, Ist. Naz. Alta Mat. Francesco Severi, Rome, 1983. MR748865 (85h:32055)

[13] N. Korevaar and R. Schoen, Sobolev spaces and harmonic maps for metric space targets. Comm. Anal. Geom. 1 (1993), no. 3-4, 561-659. MR1266480 (95b:58043)

[14] J. Petitot, The neurogeometry of pinwheels as a sub-Riemannian contact structure, J. Physiology 97 (2003), 265-309.

[15] J. Shatah, Weak solutions and development of singularities of the SU(2) $\sigma$-model, Comm. Pure and Appl. Math. 41 (1988), 459-469. MR933231 (89f:58044)

[16] J. Shatah and M. Struwe, Geometric wave equations, CIMS Lecture Notes, 2, Courant Institute of Mathematical Sciences, New York; Amer. Math. Soc., Providence, RI, 1998. MR1674843 (2000i:35135) 
[17] J. Shatah and C. Zeng, Constrained wave equations and wave maps, Comm. in Math. Phys. 239 (2003), 383-404. MR2000924 (2004g:58037)

[18] H. Sussmann, Geometry and optimal control. Mathematical control theory, 140-198, Springer, New York, 1999. MR1661472 (99k:49051)

Department of Mathematics, University of Arkansas, Fayetteville, Arkansas 72701

E-mail address: lcapogna@comp.uark.edu

Courant Institute of Mathematical Sciences, 251 Mercer St., New York, New York 10012

E-mail address: shatah@cims.nyu.edu 